\title{
Success Case of the Application of Virtual High Throughput Screening Against Molecular Antimalarial Targets
}

\author{
Renata R. Nunes, Rafael C. R. Chagas, Fernado P. Varotti \& Alex G. Taranto
}

\section{Introduction}

Malaria is an infectious disease caused by parasites belonging to the genus Plasmodium, having five species which infect humans: Plasmodium falciparum, P. vivax, P. malariae, P. ovale and P. knowlesi. ${ }^{1}$

The main symptoms presented by the disease are fever, chills, headache, vomiting, anemia, diarrhea, anorexia, fatigue. The untreated malaria can evolve to pulmonary edema, renal complications, obstructive jaundice, causing the death of infected individual. ${ }^{2}$

According to the World Health Organization (WHO), about three billion people are exposed to the risk of having malaria, of these, 216 million are affected by the disease and 655,000 die from it. In Brazil, the most important species are P. vivax, responsible for $90 \%$ of the cases, and P. falciparum, responsible for the most serious cases and for the mortality increase. A major factor contributing to the spread of the disease is the resistance of parasites to current antimalarial drugs used in therapy. ${ }^{3}$

In this context, new groups started to develop strategies to search for new antimalarial drugs. Based on molecular biology techniques and high throughtput screening. 4

Although many studies have focused on the screening of new molecules with in vitro and in vivo antimalarial activity, few compounds reached the stage of clinical trials. Therefore, it is of fundamental importance to focus efforts to find new molecules with potential antimalarial activity to create a therapeutic arsenal. ${ }^{3}$

A major factor contributing to the spread of the disease is the resistance of parasites to current antimalarial drugs used in therapy. ${ }^{3}$ In addition, the plasmodium resistance to commercially available drugs for antimalarial therapy is a threatening factor in controlling the disease worldwide. ${ }^{5}$

One strategy used for the development of new drugs is the use of in silico techniques due to high experimental costs such as X-ray crystallography and in vivo biological assay for few molecular targets. Hence, molecular modeling techniques, such as comparative modeling ${ }^{6}$, docking ${ }^{7}$, molecular dynamics $^{8}$ and virtual screening ${ }^{9}$, have been used as a tool to development of new drugs. Such techniques allow researchers to build molecular target scaffolds to simulate and predict toxicity, activity, bioavailability and effectiveness. Therefore this rational drug design project, reduces the time and the costs to develop a new drug, wherein, virtual screening approaches, consists in the identification of novel molecules against specific molecular targets. ${ }^{10}$ It has been largely used to obtain the pharmacoforic conformation and the binding energy, of a set of compounds against a biological receptor. ${ }^{11}$

In this context, our research group has studied specific targets building a database denoted by Our Own Molecular Targets (OOMT) $)^{12}$ with 40 structures 
from Protein Data Bank (PDB) ${ }^{13}$ and built by comparative homology modeling. ${ }^{6}$

In a previous study, we performed virtual screening process on 10 compounds using OOMT database. Following, the compound I showed specificity for the malaria targets. Hence, this compound was addressed for antimalarial assay. As a result of experimental work, the compound I had a satisfactory antimalarial activity. In this study, we described a success case of a hit compound obtained from virtual High Throughput Screening (vHTS). This process consisted the use of docking approach between the compound I and specific P. falciparum molecular targets, such as plasmepsin IV, plasmepsin II, falcipaina II14 and PfATP6. ${ }^{15}$

All these proteins are present in the digestive vacuole of P. falciparum, except PfATP6 present in membrane. Additionally, the digestive vacuole enzymes work with optimum $\mathrm{pH}$ of $4-5 .{ }^{16}$

\section{Methods}

The previous results of virtual screening using OOMT database motivated us to study new molecular targets against selected P. falciparum targets. The molecular targets plasmepsin IV plasmepsin II, falcipain II, which were obtained from PDB under codes 2ANL, 1LF3, 3BPF, respectively; ${ }^{13}$ while PfATP6 was obtained by previous comparative modeling methodology. ${ }^{15}$

The promising compound was designed in MarvinSketch program where its protonation was adjusted to $\mathrm{pH} 4.5$. Next, it was refined in the MOPAC17 program using the semi-empirical parametric method 7 (PM7). ${ }^{18}$

The compound was oriented toward the binding site through a grid box with dimensions of $20 \AA$ covering all binding site. The coordinates $\mathrm{X}, \mathrm{Y}$ and $\mathrm{Z}$ were defined according to table 1 with spaced points of $1 \AA$ centered in the ligand. Following, crystallographic ligands were re-docked into the targets to evaluate the docking methodology, obtaining the root mean square deviation (RMSD) values for heavy atoms. Two distinct approaches were used, rigid and flexible docking using programa AutoDock Vina7. After the rigid docking, the binding site amino acid residues were chosen for flexible docking (table 2).
Table1. Grid box size and position for all molecular targets.

\begin{tabular}{|c|c|c|c|}
\hline \multicolumn{4}{|c|}{ Coordenates ( $\AA$ ) } \\
\hline & $\mathrm{x}$ & $\mathrm{y}$ & $\mathrm{z}$ \\
\hline $2 \mathrm{ANL}$ & 54.924 & 13.448 & 25.686 \\
\hline $1 \mathrm{LF} 3$ & 16.215 & 6.850 & 27.605 \\
\hline $3 \mathrm{BPF}$ & -36.87 & 31.066 & -47.069 \\
\hline PfATP6 & -5.142 & -48.212 & 8.979 \\
\hline
\end{tabular}

In addition, the targets state of protonation was adjusted to acid $\mathrm{pH}$ using PROPKA from Maestro software ${ }^{19}$.

All docking simulations were carried using AutoDock vina ${ }^{7}$, DockThor ${ }^{20}$ and SwissDock ${ }^{21}$ softwares. The exhaustiveness was set to 8 to improve the docking search.

Finally, DS Visualizer v.4.0 (Accelrys Software

Table 2. Flexible residues in the binding sites of molecular targets

\begin{tabular}{|c|c|c|c|}
\hline 2ANL & 1LF3 & 3BPF & PfATP6 \\
\hline ASP34 & ILE14 & GLN36 & ILE251 \\
\hline GLY36 & MET15 & CYS42 & LEU253 \\
\hline ILE75 & ILE32 & TRP43 & PHE254 \\
\hline TYR77 & ASP34 & LEU72 & GLN257 \\
\hline GLY78 & GLY36 & ASN81 & LEU258 \\
\hline LEU131 & TYR77 & GLY82 & ILE261 \\
\hline ASP214 & VAL78 & GLY83 & ILE748 \\
\hline THR217 & SER79 & LEU84 & ILE752 \\
\hline VAL292 & ILE123 & HIS174 & ASN755 \\
\hline ILE300 & TYR192 & & ILE756 \\
\hline & ASP214 & & VAL759 \\
\hline & SER218 & & PHE763 \\
\hline & & & LEU815 \\
\hline & & & ILE816 \\
\hline & & & LEU821 \\
\hline & & & TYR824 \\
\hline & & & ILE825 \\
\hline
\end{tabular}


Inc, USA) was used to show the docking results of the binding conformations; thereby establishing the best molecular target for the compound. Moreover, $\log$, Molecular Weight (MW), number of hydrogen atoms acceptors and donor, Log S, Druglikeness, properties were calculated using DataWarrior program.

\section{Results and discussion}

Initially, the re-docking using AutoDock Vina process showed that the crystallographic and docked ligand shared the same conformation into the binding site. The RMSD values are represented in table 3 .

Table 3. Root mean square deviation (RMSD)

\begin{tabular}{|c|c|c|c|c|}
\hline \multirow{2}{*}{ Software } & \multicolumn{4}{|c|}{ Molecular targets - RMSD values $(\AA)$} \\
\hline & 2 ANL & 1 LF3 & 3 BPF & PfTP6 \\
\hline AutoDockVina & 0.25 & 0.40 & 1.51 & 1.12 \\
\hline DockThor & 7.09 & 2.37 & 1.59 & 3.46 \\
\hline SwissDock & 9.16 & 2.94 & 1.89 & 3.04 \\
\hline
\end{tabular}

values found after crystallographic ligands were re-docked into the targets to evaluate the docking methodology

These results evaluated the docking methodology considering the RMSD value less $2.0 \AA$. In this context, the AutoDock Vina program showed the best RMSD results compared with SwissDock and DockThor softwares7.

The AutoDock Vina ${ }^{7}$ DockThor ${ }^{20}$ and SwissDock21 software were used to generate the binding energy of compound for four enzymes. Table 4 shows the binding energies between the promise ligand and all targets for rigid and flexible approach.
Table 4. Binding energy (Kcal/mol) between the compound and binder crystallographic against Plasmepsin IV, Plasmepsin II, Falcipain II and PfATP6, using the software AutoDock Vina (Flexible and rigid), DockThor and SwissDock. Table A, B, C and D, respectively

(A)

\begin{tabular}{|c|c|c|c|c|}
\hline \multicolumn{5}{|c|}{ Flexible Docking using AutoDock Vina } \\
\hline & 2ANL & $1 \mathrm{LF} 3$ & $3 \mathrm{BPF}$ & PfATP \\
\hline Compound & -10.1 & -10.4 & -8.0 & -12.2 \\
\hline Crystal & -12.7 & -12.1 & -8.1 & -6.8 \\
\hline
\end{tabular}

(B)

\begin{tabular}{|c|c|c|c|c|}
\hline \multicolumn{5}{|c|}{ Rigid Docking using AutoDock Vina } \\
\hline & 2ANL & $1 \mathrm{LF} 3$ & $3 \mathrm{BPF}$ & PfATP \\
\hline Compound & -8.1 & -8.6 & -6.7 & -8.6 \\
\hline Crystal & -12.5 & -17.9 & -6.8 & -7.7 \\
\hline
\end{tabular}

(C)

\begin{tabular}{|c|c|c|c|c|}
\hline \multicolumn{5}{|c|}{ Using Docking DockThor } \\
\hline & 2ANL & 1 LF3 & 3BPF & PfATP \\
\hline Compound & -30.96 & -47.08 & -23.38 & -16.39 \\
\hline Crystal & -31.22 & -37.96 & -31.79 & -20.84 \\
\hline
\end{tabular}

(D)

\begin{tabular}{|c|c|c|c|c|}
\hline \multicolumn{5}{|c|}{ Using Docking SwissDock } \\
\hline & 2ANL & $1 \mathrm{LF} 3$ & $3 \mathrm{BPF}$ & PfATP \\
\hline Compound & -8.18 & -8.36 & 7.50 & -6.62 \\
\hline Crystal & -9.16 & -11.33 & -7.25 & -6.98 \\
\hline
\end{tabular}

As can be seen, the Autodock Vina program obtained more accuracy results than other softwares. In addition, both rigid and flexible approaches through AutoDock Vina suggested the 3BPF and PfATP6 as molecular targets for compound I.

The compound could perform electrostatic, van der Waals and Pi interaction. Fig. 1 shows the interaction of compound with 3BPF3 and PfATP6 into the binding site. The crystallographic ligand and compound shared the same amino acids in the binding site Fig.2.

In addition, the compound was evaluated against Lipinski rule $(\log \mathrm{P}<5$, number of hydrogen bond groups acceptors $(\mathrm{HBA})<10$,

number of hydrogen bonds groups donors (HBD) $(<5 \text { and } \mathrm{MW}<500)^{22}$ using Data Warrior software. 
As a result, the compound respects the Lipinski rule having MW, HBA, HBD, and $\log P$ of $291.377 \mathrm{~g} / \mathrm{mol}$, 4, 2 and 3.33, respectively. Moreover, this molecule has aqueous solubility and druglikeness of $-3.205 \mathrm{~mol} /$ liter and 2.76, respectively. These results are close with antimalarial drugs, like chloroquine. Furthermore, Datawarrior could not estimate any mutagenic, tumorgenic, irritant activity.

Figure 1. Electrostatic interactions, van der Waals and Pi interaction between target and compound. a) falcipaina II. b) PfTPA6. Green and pink show van der Waals interactions and electrostatic, respectively.

A)

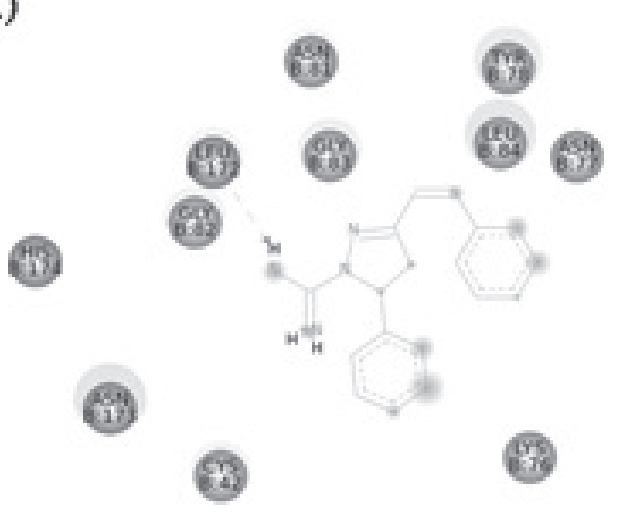

B)
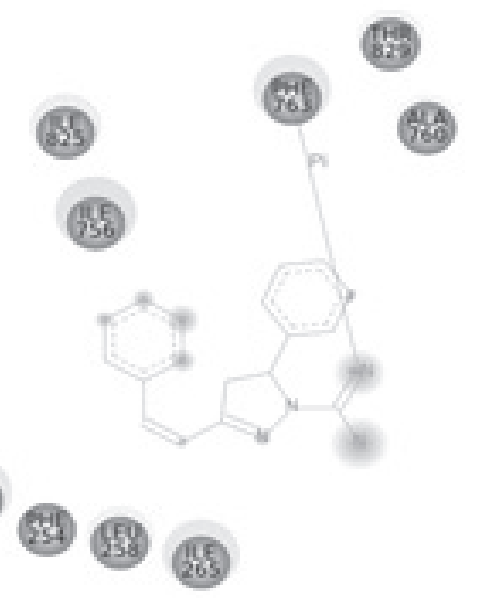

A)

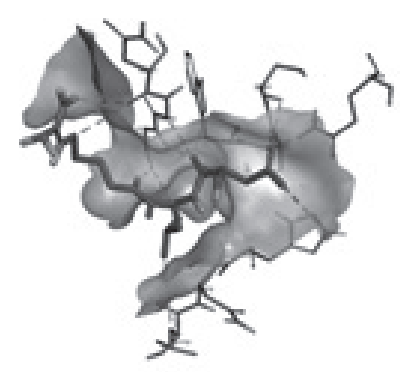

I

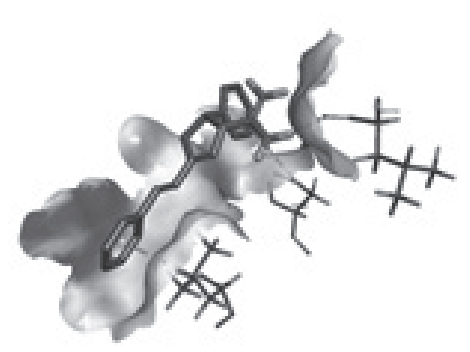

II

B)

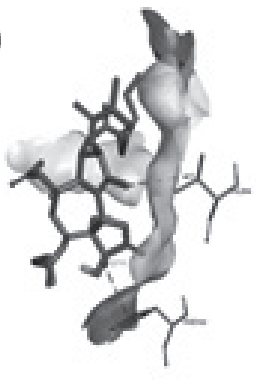

I

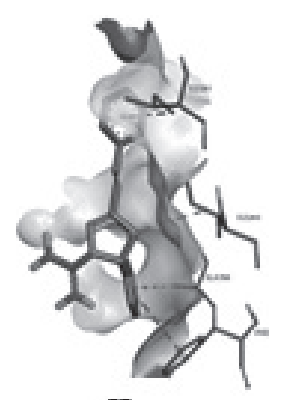

II
Figure 2. Binding site kept a) falcipain II; I - Crystallographic ligand and II - compound b) PfATP6; I - crystallographic ligand and II -compound.

\section{Conclusions}

This work evaluated the accuracy among three different docking methodologies, which AutoDock Vina showed more suitable results for our system.

The data addressed PfATP6 and falcipain II as a 
potential molecular target for this synthetic compound. In addition, this compound fits into Lipinski rule with acceptable values of $\log \mathrm{S}$ and druglikeness, suggestion it as a potentianl new antimalarial drug. Noteworthy, after docking studies, this compound was addressed to antimalarial assay. As a result, this compound was able to kill $78 \%$ of parasites in vitro test. Further ligand optimization cycle had begun generating new hit for docking and biological assay.

\section{Acknowledgments}

The authors are grateful for the support given from FAPEMIG (APQ-00557-14), CAPES, CNPq (UNIVERSAL 449984/2014-1) and PPGCF/UFSJ.

\section{References}

1. Lamikanra, A. A. et al. Blood 110, 18-28 (2007).

2. Leite, F. H. A. et al. BBR - Biochem. Biotechnol. Reports 2, 59 (2013).

3. Ferreira, M. U., M. D. S.-N. \& Silva-Nunes, M. Da. Journal of Infection in Developing Countries 4, 533-545 (2010).

4. Sahu, N. K., Sahu, S. \& Kohli, D. V. Chem. Biol. Drug Des. 71, 287-297 (2008).

5. Melo, G. C. et al. PLoS One. 9, e105922 (2014).

6. Bordoli, L. et al. Nat. Protoc. 4, 1-13 (2009).

7. Trott, O. \& Olson, A. J. J. Comput. Chem. 31, 455-461 (2010).

8. Fonseca. A. L., Nunes. R. R., Ricardo José Alves. R. J. et al. BBR - Biochem. Biotechnol. Reports 2, 111-113 (2013).

9. Westermaier, Y., Barril, X. \& Scapozza, L. Methods 71, 44-57 (2015).

10. Irwin, J. J., Sterling, T., Mysinger, M. M., Bolstad, E. S. \& Coleman, R. G. Journal of Chemical Information and Modeling (2012).

11. Andricopulo, A. \& Ferreira, L. Medicinal. J. Mod. Med. Chem. 2, 20-31 (2014).

12. Carregal, A. P., Jr, M. C. \& Taranto., A. G. BBR - Biochem. Biotechnol. Reports 2, 14-16 (2013).

13. Berman, H. M. et al. Nucleic Acids Res. 28, 235-242 (2000).

14. De Oliveira, M. E. et al. Molecules 18, 15276-15287 (2013).

15. Guimarães, D. S. M. et al. Mem. Inst. Oswaldo Cruz 110, 255-8 (2015).

16. Kuhn, Y., Rohrbach, P. \& Lanzer, M.. Cell. Microbiol. 9,
1004-1013 (2007).

17. Stewart, J.J.P., S. C. C. MOPAC2012. (2012). at <HTTP:// openmopac.net>

18. Stewart, J. J. P..J. Mol. Model. 19, 1-32 (2013).

19. Salam, N. K., Adzhigirey, M., Sherman, W. \& Pearlman, D. A. Protein Eng. Des. Sel. 27, 365-374 (2014).

20. De Magalhães, C. S., Almeida, D. M., Barbosa, H. J. C. \& Dardenne, L. E. Inf. Sci. (Ny). 289, 206-224 (2014).

21. Grosdidier, A., Zoete, V. \& Michielin, O. Nucleic Acids Res. 39, 270-277 (2011).

22. Jorgensen, W. L. Science 303, 1813-1818 (2004).

\section{Renata R. Nunes*, Rafael C. R. Chagas, Fernado de P. Varotti \& Alex G. Taranto}

Universidade Federal de São João del Rei - Campus CCO - Rua Sebastião Gonçalves Coelho, 400 - Chanadour - MG - CEP 35.501-296 *E-mail: renata.rachidee@gmail.com 